\title{
Ictal semiology, functional anatomy and multimodal diagnostic in patients with insular epilepsies
}

Hermann Stefan ${ }^{1 *}$ and Stephanie Gollwitzer ${ }^{2}$

\begin{abstract}
Background: The insula is a hidden part in the cerebral cortex. Insular epilepsy is underrecognized and it bears a special risk for misdiagnosis with regard to nonepileptic seizures or wrong localization of epileptic seizures.

Case presentations: The manuscript describes 2 cases with ictal semiology of paresthesia and pain followed by hyperkinetic movements, noninvasive findings of source localization and/or invasive SEEG exploration.

Conclusion: Magnetic source imaging, ictal SPECT as noninvasive and invasive recordings with depth electrodes (SEEG) can provide important preoperative information for the involvement of insular and periinsular regions in focal pharmacoresistant epilepsies. The optimal use of these methods presupposes extensive knowledge of ictal semiology and other clinical characteristics. The clinical localization hypothesis can be optimally proofed by SEEG exploration.
\end{abstract}

Keywords: Insular epilepsy, Ictal semiology, Source localization, MEG/EEG, SEEG

\section{Introduction}

Insular epilepsy bears a special risk for misdiagnosis with regard to nonepileptic seizures or wrong localization of epileptic seizure onset. Two cases are reported with ictal semiology of paresthesia and pain followed by hyperkinetic movements and results of noninvasive recordings including findings of source localization and/or invasive SEEG exploration.

\section{Case presentations}

Case 1

History and examination

Twenty-one year old male patient with focal epilepsy, one seizure per month.

\section{Neurological findings}

Normal, right handed.

\section{Video-EEG}

EEG interictal Spikes $100 \%$ in the right hemisphere right shown in electrodes F6, FC6, C6, F8, TP8, T8.

\footnotetext{
* Correspondence: hermann.stefan@t-online.de

'Department of Neurology, Biomagnetism, University Hospital Erlangen, 10,

Schwabachanlage, D, -91054 Erlangen, Germany

Full list of author information is available at the end of the article
}

Ictal signs Paresthesia left hand, hyperkinetic seizures, extension of the left arm and flexion of the right leg, with head body movements and dystonia of the left arm (Fig. 1).

EEG ictal Beta and muscle activity frontal right shown in electrodes FC4, C4, F4, FC6, C6, F6. During five seizures flattening of background activity in EEG bilateral. Five seizures showed no seizure pattern.

\section{MRI}

FCD right frontal pars opercularis and insula (Fig. 2).

\section{Spectroscopy}

Decrease of NAA inside of dysplasia frontal right in comparison to the opposite.

\section{Neuropsychology}

Deficit of object identification and executive function (hemispherical fronto parietal dysfunction right).

\section{Ictal SPECT}

Subtraction analysis: Localization of hyperperfusion insula right (Fig. 3). 


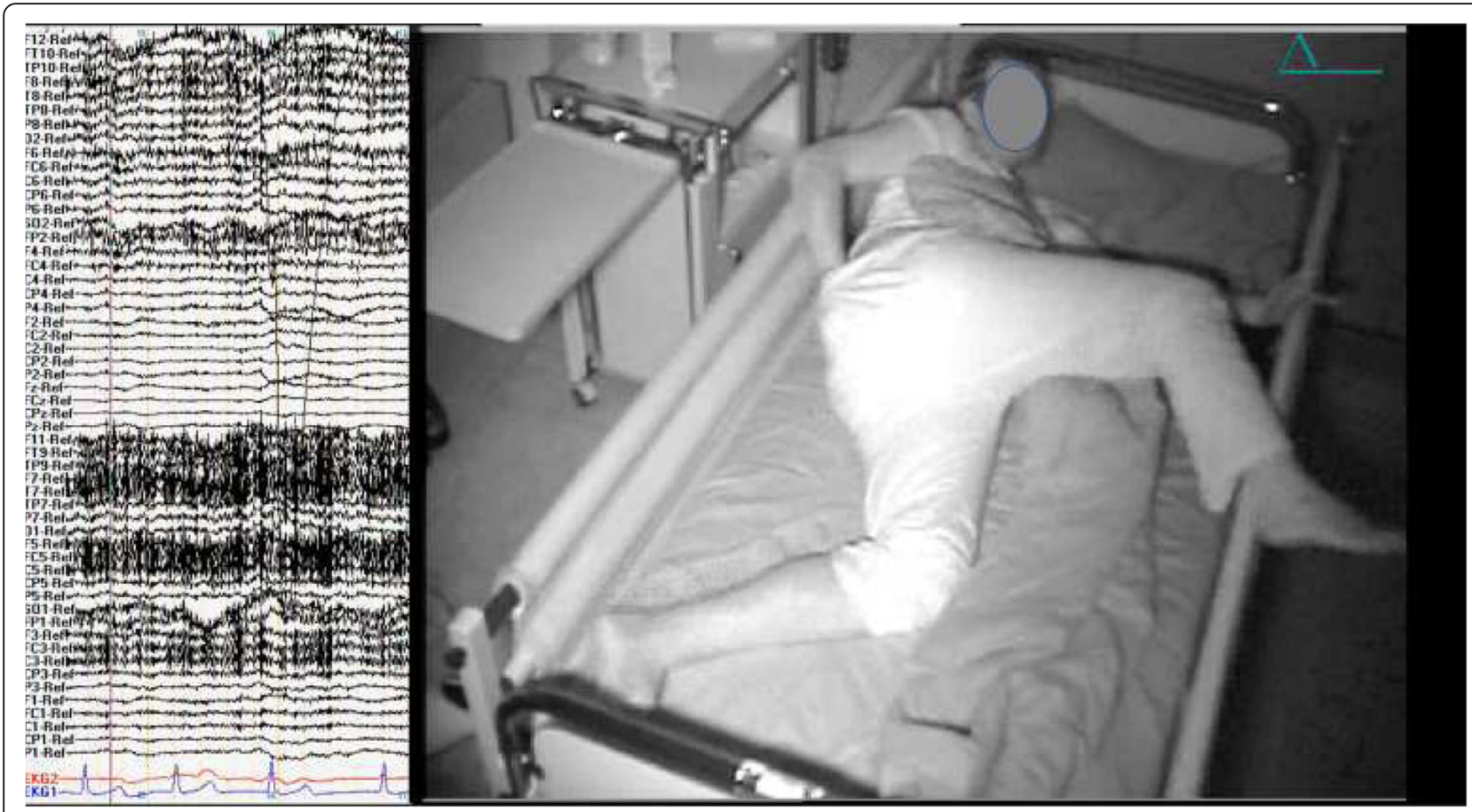

Fig. 1 During the seizure with hyperkinetic movements the ictal EEG shows no epileptiforme activity but movement artifacts

\section{MEG}

dSPM localization insula right (Fig. 4).

Comment Insular seizures should be considered and differentiated from pseudoepileptic psychogenic attacks when hyperkinetic seizure signs are combined with missing epileptiform seizure activity in surface EEG. Background flattening is a common pattern of ictal in extratemporal lobe originating seizures. EEG Source localization of interictal spike activity in MEG can point to the insular region. In addition to dipole analysis another approach to compensate for depth bias is the noise-normalized dynamic statistical parametric map (dSPM) technique. Ictal sensory signs can be difficult to differentiate from primary somatosensory cortex in the parietal lobe. From the parietal cortex spread of ictal activity to insula or frontal lobe can be associated with hyperkinetic movements.

Ictal SPECT offers the possibility of lobar confirmation if the injection of the radioligand is performed with a short latency after seizure onset. Here a semiautomatic injection device can be used. Invasive video-EEG recordings are necessary to delineate the seizure onset in the opercular-insular region.

The seizures of this patient were markedly reduced by changing antiepileptic drug treatment. In patients unsuccessfully taking three or more antiepileptic drugs in the longterm course of the treatment, epochs with improvement of seizure frequency or severity can occur. After the preoperative evaluation only focal aware seizures (one per month) with paresthesia in the left hand persisted. Therefore the patient gave no consent for further invasive investigations and epilepsy surgery was postponed.

\section{Case 2}

\section{History and examination}

Male patient, suffering from epilepsy since the age of 14. His habitual seizures began with a tingling sensation in his right thigh followed by pain over his whole body. He then usually lost awareness and automatisms including lip smacking and body rocking were regularly observed. The epilepsy turned out refractory to medical treatment. There was no history of febrile convulsions, CNS infections, head trauma or perinatal complications, his family history was unremarkable.

\section{Neurological examination}

Normal, right handed.

\section{MRI}

Cranial MRI revealed no pathology.

\section{Neuropsychological examination}

No memory deficits. 


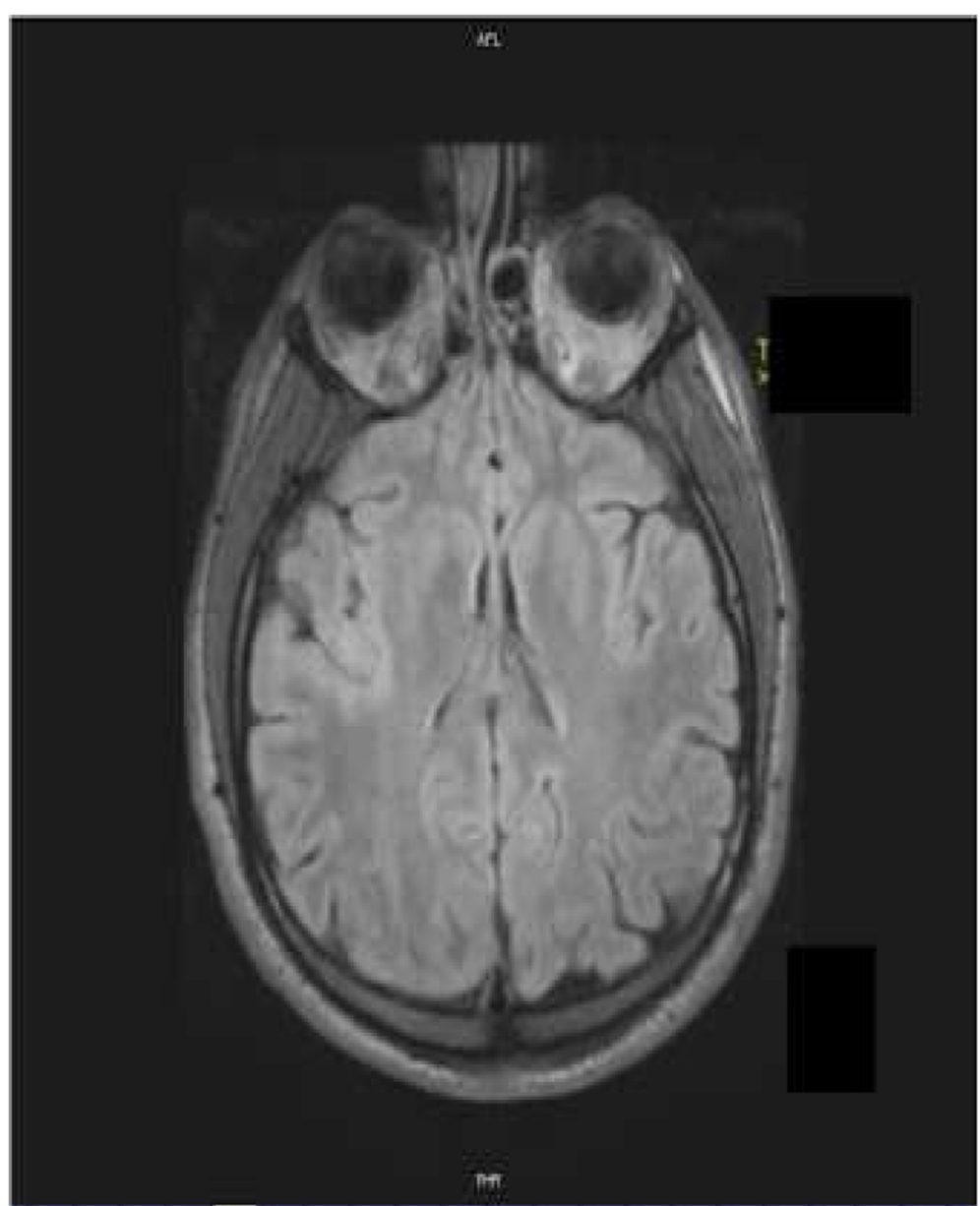

Fig. 2 MRI lesion in insula right and pars opercularis

\section{Video EEG}

Surface EEG Interictal scalp EEG demonstrated frequent regional left temporal sharp waves. Nine habitual seizures were recorded. In all cases a left temporal theta seizure pattern was visible but appeared late up to $50 \mathrm{~s}$ after clinical seizure onset (Fig. 5).

MEG showed spikes in the left insular-temporal region (Fig. 6). Based on semiology and non-invasive findings an invasive EEG study with stereotactically implanted depth electrodes was designed.

Invasive EEG 12 depth electrodes were implanted. The implantation scheme targeted mainly the right insula and the right temporal lobe (Fig. 7) SEEG evaluation revealed frequent spikes in the left posterior insula. Intermittent high amplitude spikes were seen in mesial temporal structures. The frequency of temporal spikes increased during withdrawal of anticonvulsants. Five habitual seizures were recorded. In all cases the patient reported the typical tingling sensation in his right thigh followed by pain all over his body. He then showed impaired awareness and automatisms. SEEG onset consisted of a DC shift followed by low voltage fast activity in the left superior posterior insula (electrode LPc contact 5) and consecutive electrodecrement in the inferior posterior insula (electrode LTc contact 1 ) followed by rhythmic spikes eventually evolving to a rhythmic pattern in the alpha frequency range remaining circumscribed in this region (Figs. 8 and 9). Cortical stimulation was delivered to posterior insular contacts eliciting the patient's typical aura.

Comment In this case the stereotypical semiology and especially the rare phenomenon of ictal pain in the thigh indicated insular epilepsy. The first sensory ictal sign (tingling) occurred in the thigh followed by generalized pain sensations. These symptoms including hyperkinetic movements may lead to a first glance of a wrong diagnosis of psychogenic nonepileptic pseudoseizures. If only pain exists during a focal aware seizure and EEG shows no epileptiform activity the correct diagnosis may be missed for years. Ictal sensory signs including pain in the thigh are typical insular signs. Whereas surface EEG only showed ictal epileptic activity in the posterior 


\section{MEG}

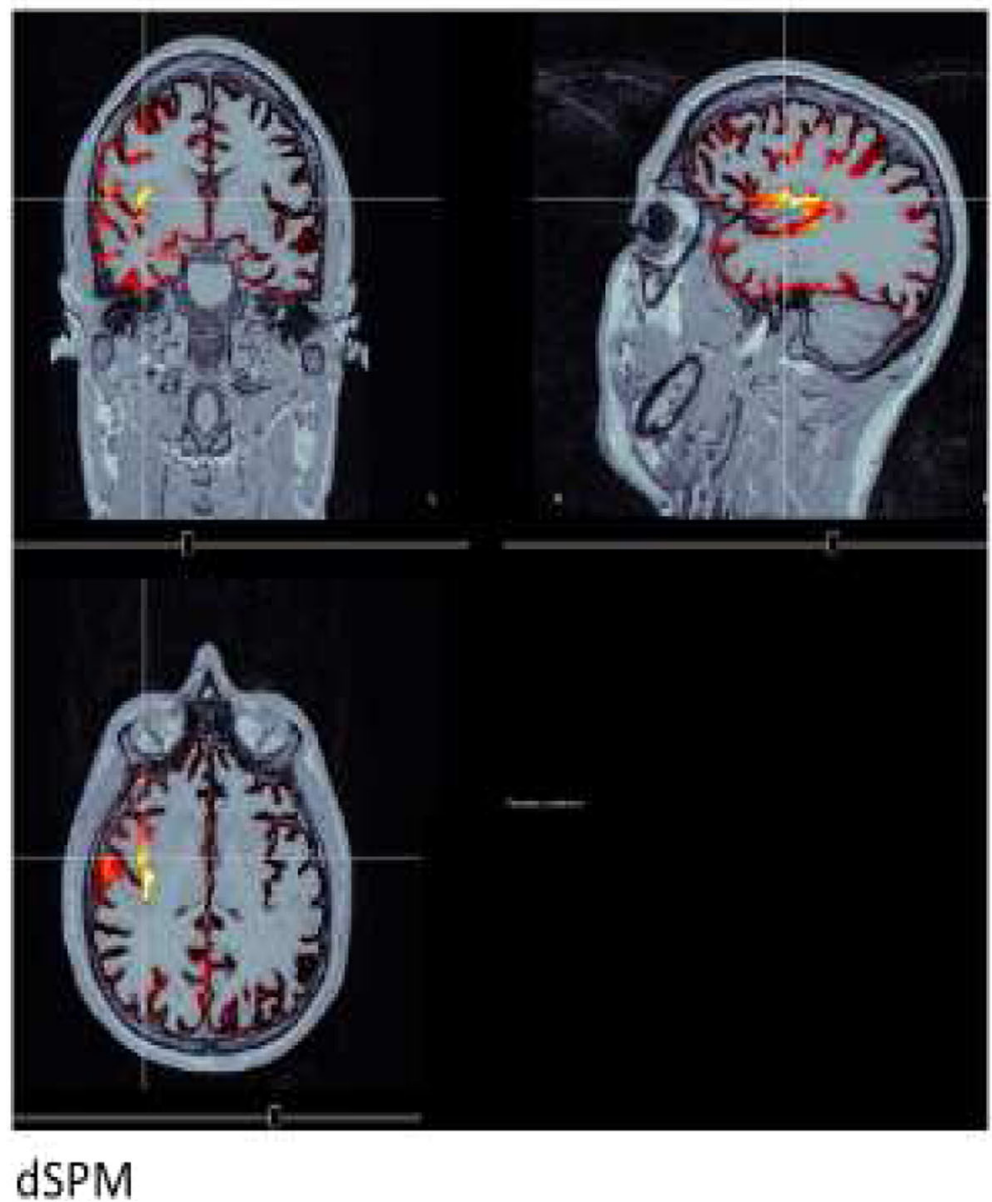

Fig. 3 Left: Ictal EEG with muscle artefacts, tachycardia after seizure onset. Injection of technetium HMPAO $5 \mathrm{~s}$ after seizure onset for Single Photon Emission Computed Tomographn (SPECT). Right: SPECT demonstrates total hyperfusion in the sylvian region (courtesy Prof. T. Kuwert, University Hospital Erlangen)
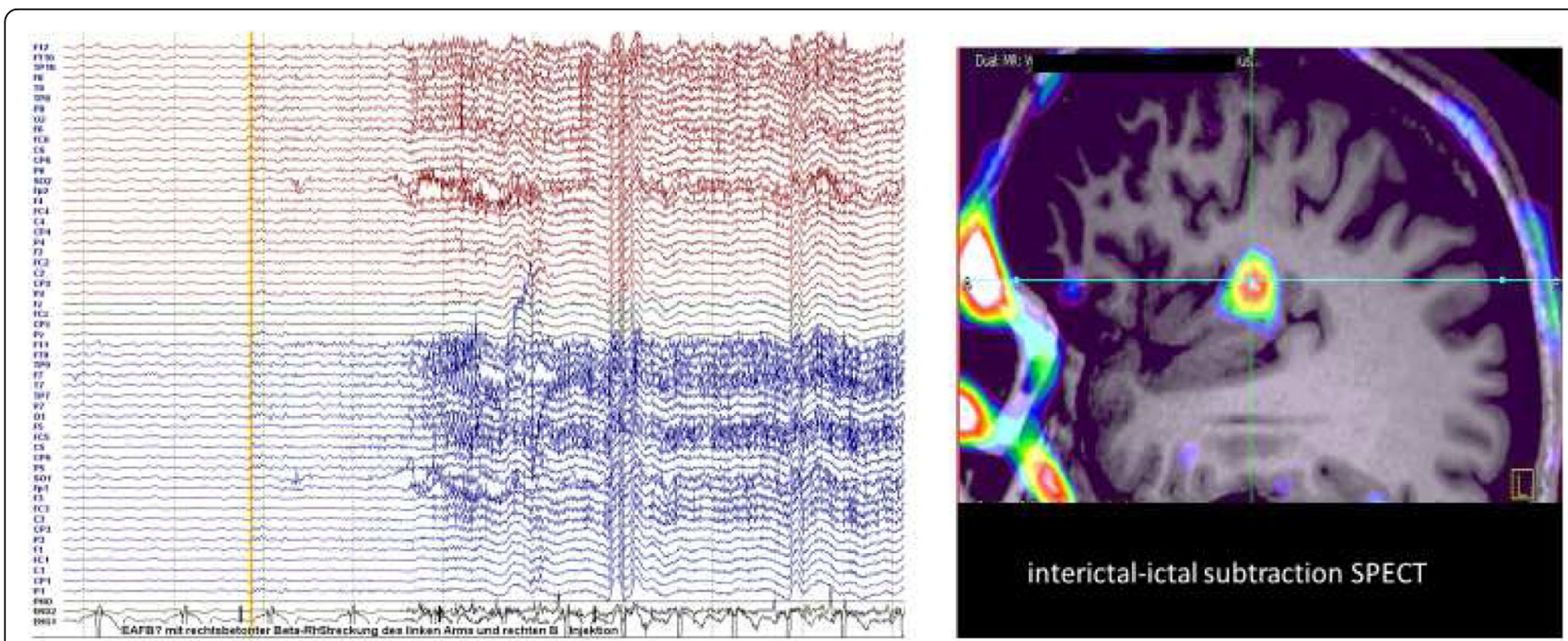

Fig. 4 MEG localization in the insula (yellow); dynamic statistical parametric maps (dSPM) 


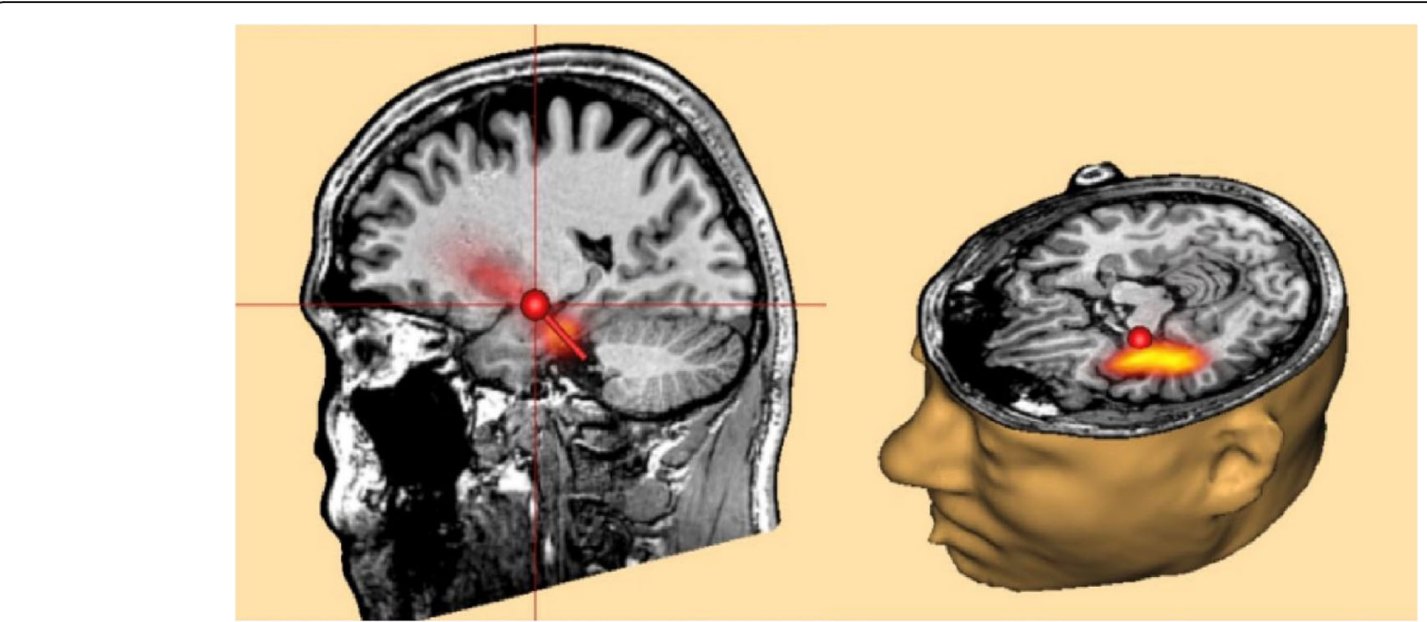

Fig. 5 Dipole and Clara localization Dipol- und Cara-localisation from 165 MEG-spikes (average, RV 3,8\%), focal noninvasive interictal localization points to insular-temporal left

temporal surface electrodes MEG pointed to the insular region. Therefore depth electrodes were implanted there. Invasive EEG confirmed the hypothesis of insular epilepsy and lead to the localization of the seizure onset zone in the left superior posterior insula. Despite normal MRI the patient was offered a circumscribed resection of the upper posterior insula.

The reported two cases stimulate a short updated insight into current knowledge of functional and clinical aspects of the insular system.

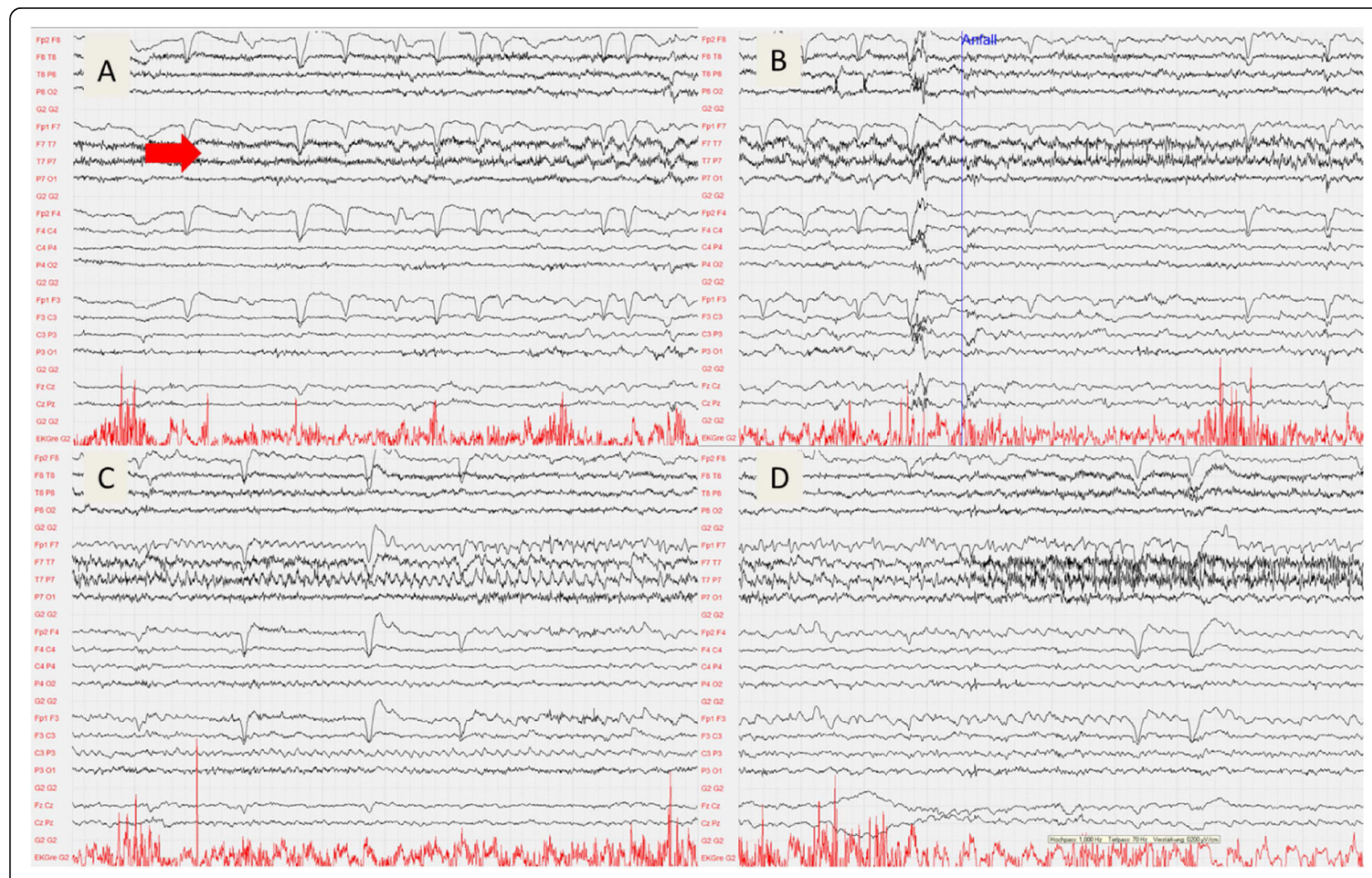

Fig. 6 Ictal scalp EEG. A left temporal seizure pattern appeared up to $50 \mathrm{~s}$ after clinical seizure onset 

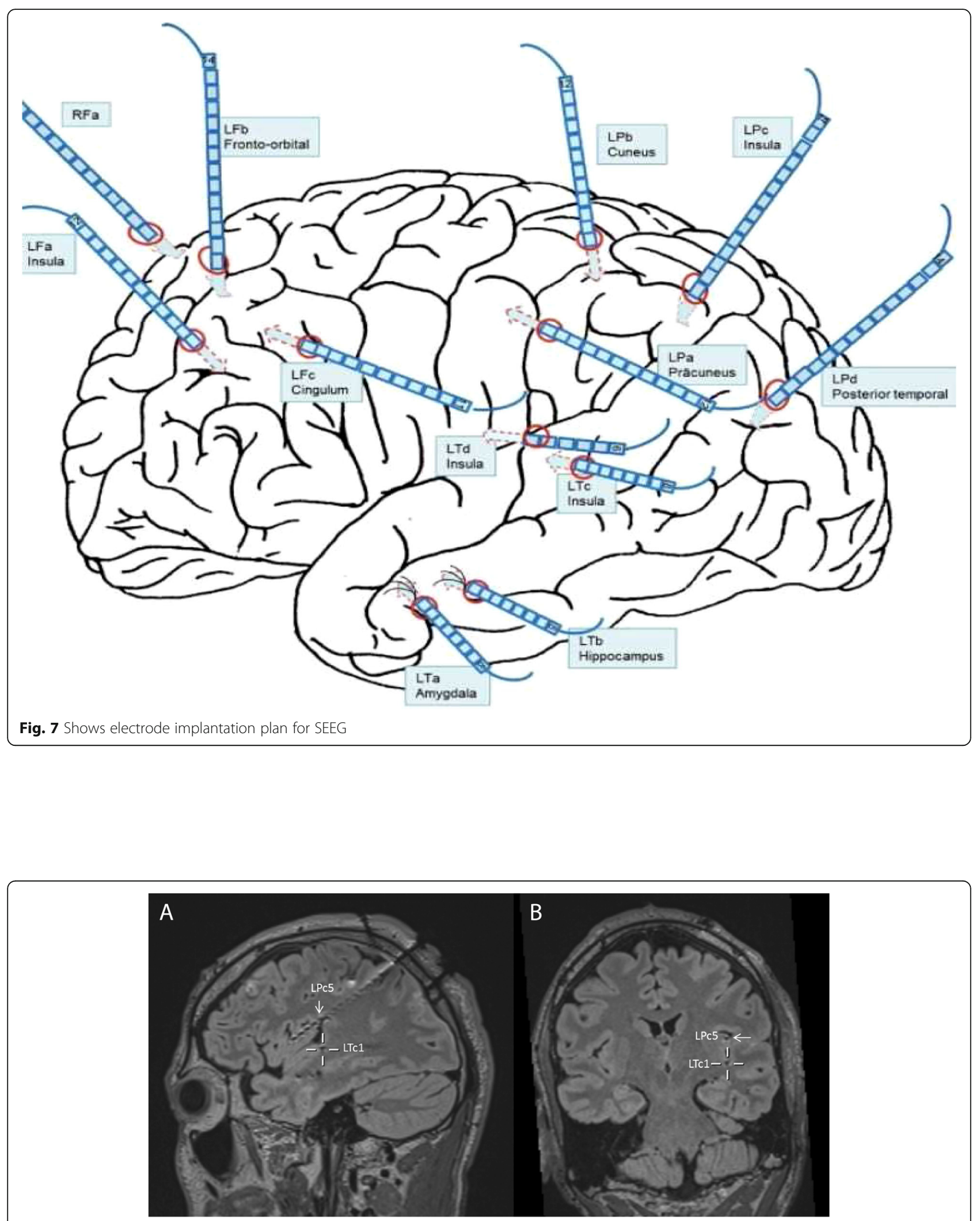

Fig. 8 Location of sEEG electrodes involved in seizure onset, as demonstrated by postoperative MRI scan (FLAIR sequence). a sagittal image depicting whole length of electrode LPC and contact 1 of electrode LTC. $\mathbf{b}$ coronal view showing contact 5 of electrode LPC and contact 1 of elecrode LTC 


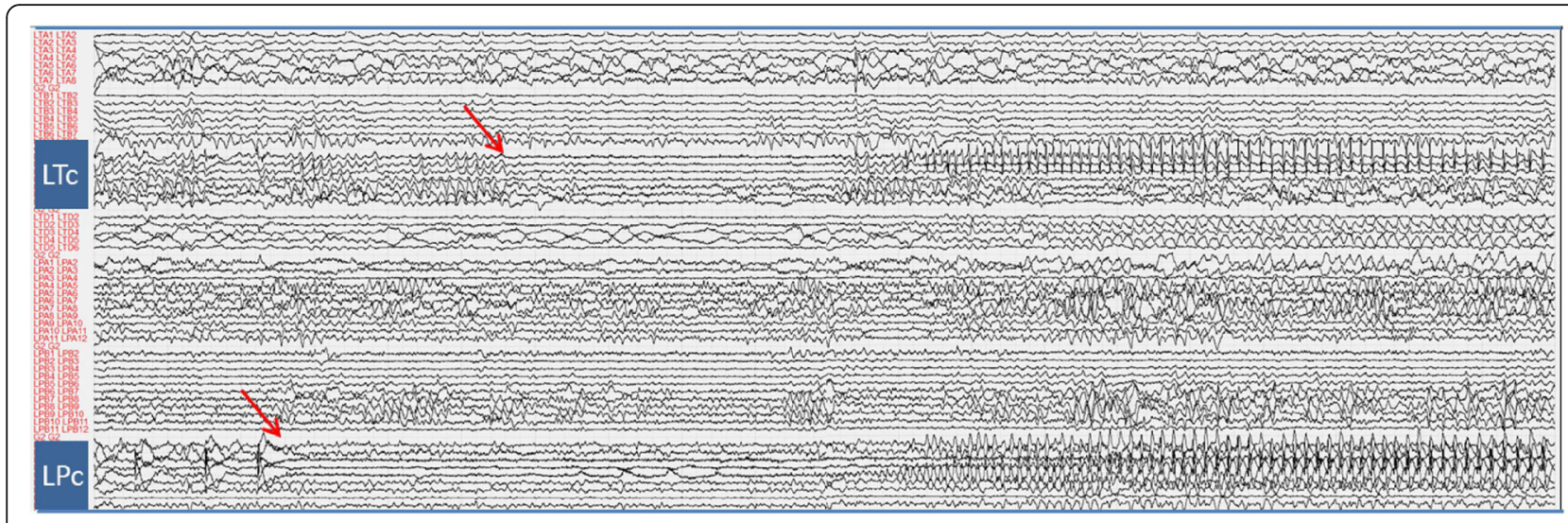

Fig. 9 SEEG ictal recording showing ictal onset in LPC (superior posterior insula) and early seizure spread to LTC (inferior posterior insula)

\section{Function and clinical characteristics of the insula in relation to the cases}

Johannes Reil (1759-1813) working in Halle and Berlin described the island (Insula Reilii) 1796 as hidden part of the folded cortex located under temporal- and frontoparietal operculum. The insula is "burried" in the fissura Sylvii (first description by Sylvius, Frans de le Boe, anatomist in Amsterdam1641). The insula is differentiated in three anatomical regions: The regio insularis agranularis, the regio insularis propea granularis and the regio insularis granularis. Insight into the function mediated by the insula was given by invasive brain stimulations.

Invasive explorations using electro stimulations of cortical regions in the sylvian fissure [1-4] demonstrated that the insula is responsible for sensory cognitive and emotional functions, in addition the insula participates in autonomic functions. Painful sensations were elicited mostly from the posterior third of the insula. This is congruent with the findings of case two with ictal pain all over the patient's body. During electric stimulation pain miss sensations only were triggered elicited by insular and secondary somatosensory cortex (SII) stimulations not by stimulating primary somatosensory cortex (SI) or other cortical regions [5]. Other functions concern empathic feeling, emotional reactions to pain, gustatory smell or hearing changes.

Invasive explorations with electrostimulations in cortical regions of the sylvian fissure [1] provoked sensations such as a feeling of sinking, nausea, pain in the navel area, rising epigastric sensations, pain ipsilateral abdominal contralateral in rib region. The insula has far reaching connections [2] with the neocortex, basal ganglia, thalamus, limbic structures and olfactory cortex. Because of rapid propagation of ictal activity of the insula to different connected lobes recorded ictal signs may be already the result of spread. Therefore difficulties do exist to distinguish whether the symptoms originate from the insular or its surrounding structures.

\section{Semiology}

For the differentiation of insular seizures with nonepileptic psychogenic seizures or other focal epilepsies the knowledge of the semiology of insular seizures is an important precondition.

Diagnostic leading ictal signs concern:

Awareness, laryngeal constriction, throat discomfort (suffocation, breathlessness as a result of tonic constriction of throat muscles), paresthesia, chest constriction, dyspnea, dysarthric speech, unpleasant abnormal sensoric sensations (case 1 and 2) like electric sensation, temperature increase or pain are characteristic. The signs may initially arise in the perioral region. From there they can spread bilaterally to extremities or other parts of the body. In addition motoric signs like hyperkinetic movements [6] (case 1 and 2), tonic contractions of eye, arc de cercle, face or extremity muscles, aphasia or dysarthria may occur. Further symptoms can be epigastric aura, gustatory sensations, hypersalivation, visceral motoric signs (vomiting, defecation urge), auditory hallucinations, sensory aphasia or autonomic such as bradycardia (left insula), tachycardia (right insula). The recognition of the ictal semiology can be hampered by the fact that seizures appear not infrequently during sleep [7-14].

Penfield and Jasper [1] pointed out that seizures originating in the insula may have a semiology similar to that of temporal lobe seizures.

Several ictal phenomena can be explained by the connectivity of different brain regions. Indirect connection parallel and lateral to the classic fasciculus arcuatus connects the Broca-region with the inferior parietal lobe. This perisylvian tract may explain ictal conduction aphasia [15] 
and focal-motoric phenomena in the face or extremities by connections to frontal lobe regions. Neuronal cerebral regions in the granular fields of the insula are large and bilaterally represented. This explains the existence of bilateral paresthesia (case 2). The second sensory area has a key position with regard to somatic informations to the limbic structures also for the tactile memory. In addition the insula has a "gate function" between somatosensoric region S1 and S2 as well as limbic structures in the temporal lobe e.g. amygdala. The temporal pole is interpreted as limbic integration cortex, which connects orbitofrontal and insular cortical functions. Laryngeal sensations and oral automatisms as well as gustatory perceptions are comprehensible by insular opercular representations for the function of ingestion. Diffusion tensor investigations (tractography) showed connections through the inferior parietal cortex.

Three spreading pathways in the epileptic system were differentiated:

1 Temporal-lateral fissure-insular lobe, mainly the insular operculum

2 Temporal-limbic system-insular lobe, inner structure of tempus and/or temporal pole.

3 Inner side-orbitofrontal-insular lobe, including the inner side of the insular lobe and the orbitofrontal cortex [16]. Insular-opercular sleep related seizures may occur with initial viscerosensitive or somatosensory symptoms and tonic-dystonic asymmetric posturing and/or hyperkinetic movements, including bimanual/bipedal activity as well as ballistic motor signs [17]. Opercular seizures may be manifested with initial laryngeal or other ictal signs typically seen in insular epilepsy, too [18].

\section{Etiology}

In case 1 an FCD is detected in MRI.

Causes mostly detected are low grade tumors (27\%), focal cortical dysplasia (FCD)(21\%), vascular malformation, cavernomas (19\%), atrophy/ gliosis (17\%) and normal tissue (8\%) [19]. Genetic defects were described in operculo-insular epilepsy cases, including mutations in the CHRNB2 and CHRNA4 genes in patients with sleep-related hyperkinetic seizures [20].

In a family with reflex bathing epilepsy a temporoinsular epileptogenic network was reported with a Q555X mutation in synapsin 1 on chromosome Xp11-q21 [21].

\section{Imaging}

In case 1 an extended FCD right frontal in pars opercularis and insula is shown by structural MRI.

MR-Spectroscopy detects a decrease of NAA inside of the dysplasia, frontal right in comparison to the opposite side. In case 2 no abnormal finding in MRI was detected. From other cases we know that MRI is very helpful in discovering subtle lesions like FCD or small tumors. Occasionally MRI also detects a secondary hippocampal sclerosis [9]. During presurgical evaluation the MRI of the operculo-insular area turned out normal or showed uncertain findings in $72 \%$ [22].

In difficult cases voxel based morphometry [23], ictal SPECT or PET [20, 24] provide additional informations.

\section{Electrophysiology \\ Surface EEG}

Surface EEG often fails to detect the insular focal epileptic activity (case 1 no epileptiforme activity, case 2 later during the seizure temporal. Ictal discharges often are missed in surface EEG. Ictal flattening can be the only surface EEG change. In case of seizure onset in the anterior insula propagated epileptiform activity may be wrongly recorded as seizure onset in frontal or temporal areas or if true seizure onset is in posterior insula parts then in central regions.

\section{SPECT, PET, MEG}

In operculo-insular epilepsy ictal SPECT correctly identified the focus in $65 \%$ and provided misleading information in $18 \%$ [25]. MEG pointed to the insula in case 1 and insular-temporal region in case 2 .

MEG source localization was superior in localizing insular focal epileptic activity compared to simultaneous surface EEG, PET and SPECT [23, 26, 27]. In the cases reported by Heers et al. [23] subtle lesions were missed in MRI. MEG and MAP permitted to proceed to presurgical evaluation using selective MEG guided invasive exploration with depth electrodes, thus epileptogenic lesions could be successfully resected.

\section{Invasive recordings}

In case 2 ictal onset only could be localized by SEEG. The scheme of electrode implantation is shown in Fig. 7. The electro-anatomic localization of ictal SEEG onset matches with the clinical localization hypothesis by ictal semiology interpretation.

If a clear location of a tumorous lesion is detected in the anterior part of the insula and ictal semiology is congruent with noninvasive diagnostics no further extensive invasive exploration may be necessary. However in other situations depth recordings in the insula and temporalor frontal lobe are recommended e.g. using stereoelectroencephalography (SEEG).

SEEG can detect simultaneous insular-opercular seizure onset in patients with sleep related paroxysmal motor behaviours [17]. In MRI normal cases extensive individual sampling also of extrainsular regions to which the insula is closely connected should be performed [28]. 


\section{Treatment}

For case 1 currently no epilepsy surgery will be carried out because of antiepileptic drug treatment seizures surprisingly are reduced after first noninvasive phase and dismissal.

Case 2 is planned for epilepsy surgery because of persisting severe impairment of quality of life by seizures. In pharmacoresistent patients resective epilepsy surgery can be performed, bearing however an increased risk of complications because of high vascularization in this area and location to eloquent cortex. Complications were reported between 8 and $45 \%$ [29]. With regard to risks resections predominantly are performed in the anterior part. Because the insula is integrated in the paralimbic network with the orbitofrontal temporopolar region, temporopolar mesial epileptic activity invades the sylvian or perisylvian region and vice versa. This may give rise to complex semiological pattern. Hidden causes of surgery-resistant temporal lobe epilepsy were differentiated as: extra temporal or temporal plus epilepsies by Ryvlin and Kahane [30]. Seizures arising from the anterior part of the insula producing visceromotor activities and may be misdiagnosed as temporomesial originating seizures [6]. Postoperative seizure outcome according to different etiologies with a follow up of 1 year was $52.2 \%$ [31].

Seizure outcome after removal of insular tumors was reported and demonstrated in $82 \%$, Engel I outcome in $45 \%$ and only transient morbidity in a series of 11 patients. The authors state that these results suggest taking account of the technical surgical progress now allow to minimize the morbidity after surgery in this region [32].

Treatment failures after resection of the temporal lobe in "temporal lobe epilepsy" may be due to missed seizure onset in the insula. In case of selective insular cortex resection it can be due to the fact that the true seizure onset was elsewhere and the insula with its manifold connections to other lobes was just involved as a relais station in the complex epileptic network.

If no resective surgery is possible then other options for treatment are radiosurgery, radiofrequency thermocoagulation [33] or laser interstitial thermal therapy ablation. LITT permits ablation of abnormal tissue while sparing normal cortex. Perry et al. [34] reported results of 20 patients which had in $70 \%$ normal MRIs. After LITT 50\% postoperative were in Engel class 1 and 5\% in class 2. Adversed functional effects (29\%) in most cases disappeared after 6 months or persisted only as minimal residual dysfunction.

Improved surgical procedures using neuronavigation, intraoperative MRI, ultrasound and approaches for minimal invasive ablation offer the possibility to improve effectiveness of surgery in insular epilepsies.

\section{Conclusion}

For difficult to diagnose and localize insular epilepsy noninvasive methods like MEG/EEG source localization including dipole, dSPM and ictal SPECT provide important information. For presurgical evaluation in addition SEEG provides further essential information.

\section{Abbreviations}

CHRNA4: Cholincergic receptor nicotinic alpha 4 submit; CHRNB2: Cholinergic receptor nicotinic beta 2 submit; CLARA localizations: Classical LORETA analysis recursively applied; CNS infections: Central nervous system infections; DC shift: Direct current shift; dSPM: dynamic statistical parametric map; EEG: Electreoencephalography; FCD: Focal cortical displasia; HMPAO: Hexamethyl propylene amine oxime; LITT: Laser interstitial thermal therapy; MAP: Morphometric analysis program; MEG: Magnetoencephalography; MRI: Magnetic resonance imaging; NAA: Nacetylaspartat; PET: Positron emission tomography;

SEEG: Stereoelectroencephalography; SPECT: Single photon emission computed tomograpgy

\section{Acknowledgements}

We wish to express our gratitude to Prof.Dr. T. Kuwert, Clinic for Nuclear Medicine, Erlangen, for providing ictal SPECT data and PD Dr. S. Rampp, Clinic of Neurology, Erlangen, for provinding MEG data as well as Prof. Dr. K. Rössler for providing data of depthelectrode implantation, Clinic for Neurosurgery, Erlangen.

\section{Author's informations}

Dr. Gollwitzer has been informed of all data an given her consent.

\section{Author's contributions}

Both authors read and approved the final manuscript.

\section{Disclosures}

Hermann Stefan received grants from Deutsche Forschungsgemeinschaft, honoraria and travel reimbursement from Elekta Oy, Helsinki, Finland. There is no conflict of interest concerning the content of this paper.

Stephanie Gollwitzer has served on the scientific advisory board or on the speaker's bureau or received grants from of Desitin, Eisai and UCB Pharma. There is no conflict of interest concerning the content of this paper.

Funding

Not applicable for that section.

Availability of data and materials

Clinic of Neurology - Epilepsy Center, Clinic for Nuclear Medicine, Clinic for Neurosurgery, Unversity Hospital Erlangen.

Ethics approval and consent to participate

Not applicable for that section.

Consent for publication

Not applicable for that section.

Competing interests

The authors declare that they have no competing interests.

\section{Author details}

${ }^{1}$ Department of Neurology, Biomagnetism, University Hospital Erlangen, 10, Schwabachanlage, D, -91054 Erlangen, Germany. ${ }^{2}$ Department of Neurology, Epilepsy Center, University Hospital Erlangen, Erlangen, Germany.

Received: 3 June 2019 Accepted: 26 August 2019

Published online: 03 December 2019

\section{References}

1. Penfield $\mathrm{W}$, Jasper $\mathrm{H}$. Epilepsy and the functional anatomy of the human brain. Boston: Little, Brown; 1954.

2. Augustine JR. Circuitry and functional aspects of the insular lobe in primates including humans. Brain Res Brain Res Rev. 1996;22:229-244.8. 
3. Ostroesky K, Magnin M, Ryvlin P, Isnard J, et al. Representation of pain and somatic sensation in the human insula: a study of responses to direct electrical cortical stimulation. Cereb Cortex. 2002;12(4):376-85.

4. Mazzola L, Mauguiere F, Isnard J. Electrical stimulations of the human insula: their contribution to the ictal semiology of insular seizures. J Clin Neurophysiol. 2017;34:307-14.

5. Ostrowsky K, Isnard J, Ryvlin P, Guénot M, et al. Functional mapping of the insular cortex: clinical implication in temporal lobe epilepsy. Epilepsia. 2000; 41:681-686.9.

6. Knight E, Pestana M, Grinenko O, Bulacio J, et al. Arc de cercle as a manifestation of focal epileptic seizures. Neurol Clin Pract. 2018;8(4):354-6.

7. Isnard J, Guénot M, Ostrowsky $K$, Sindou M, et al. The role of the insular cortex in temporal lobe epilepsy. Ann Neurol. 2000;48:614-623.36.

8. Isnard J, Guénot M, Sindou M, Mauguière F. Clinical manifestations of insular lobe seizures: a stereo-electroencephalographic study. Epilepsia. 2004:45:1079-93.

9. Nguyen DK, Nguyen DB, Malak R, Bouthillier A. Insular cortex epilepsy: an overview. Can J Neurol Sci. 2009 a;36(suppl. 2):58-62.6.

10. Nguyen DK, Nguyen DB, Malak R, Leroux JM, et al. Revisiting the role of the insula in refractory partial epilepsy. Epilepsia. 2009 b;50:510-520.7.

11. Ryvlin P, Minotti L, Demarquay G, Hirsch E, et al. Nocturnal hypermotor seizures, suggesting frontal lobe epilepsy, can originate in the insula. Epilepsia. 2006:47:755-765.41.

12. Kriegel M, Roberts D, Jobst B. Orbitofrontal and insular epilepsy. J Clin Neurophysiol. 2012;29:385-91.

13. Wang F, Xu Y, Li Z, Xu J, et al. Surgical strategies and seizure outcomes of patients with epilepsy due to insular lesions. Chin J Neurosurg. 2012; 28:392-395.15.

14. Gschwind M, Picard F. Ecstatic epileptic seizures: a glimpse into the multiple roles of the insula. Front Behav Neurosci. 2016;10:21.

15. Catani N, Jones DK, Ffytche DH. Perisylvian language networks of the human brain. Ann Neurol. 2005;57(1):8-16.

16. Sun T, Wang F, Cui J. Insular cortex and insular epilepsy. J Neurol Neurosci. 2016;1(6). https://doi.org/10.21767/2171-6625.100009.

17. Prosperio P, Cossu M, Francione $S$, Tassi L, et al. Insular-opercular seizures manifesting with sleep related paroxysmal motor behaviours. a stereo-EEG study. Epilepsia. 2011;52(10):1781-91.

18. Stefan H, Buchfelder M. Diagnostik sylvischer und perisylvischer Epilepsien Nervenarzt. 2007;78:1175-81

19. Chevrier M, Bard C, Guilbert F, Nguyen D. Structural abnormalities in patients with insular/periinsular epilepsy. Am J Neuroradiol. 2013;34:2152-6.

20. Obaid S, Zerouali Y, Nguyen DK. Insular epilepsy: semiology and noninvasive investigations. J Clin Neurophysiol. 2017;34:315-23.

21. Nguyen DK, Rouleau I, Senechal G, et al. X-linked focal epilepsy with reflex bathing seizures: characterization of a distinct epileptic syndrome. Epilepsia. 2015;56(7):1098-108.

22. Bouthillier A, Nguyen DK. Epilepsy surgeries requiring an operculoinsular cortectomy: operative technique and results. Neurosurgery. 2017;81(4):602-12.

23. Heers M, Rampp S, Stefan H, Urbach $H$, et al. MEG based identification of the epileptogenic zone in occult insular epilepsy. Seizure. 2012;21:128-33.

24. Fei $P$, Soucy JP, Obaid S, Boucher $O$, et al. The value of regional cerebral blood flow SPECT and FDG PET in operculoinsular epilepsy. Clin Nucl Med. 2018;43:e67-73.

25. Weil A, Le N, Jayakar P, et al. Medically resistant pediatric insular-opercular/ perisylvian epilepsy. Part 2: outcome following resective surgery. J Neurosurg Pediatr. 2016;18(5):523-35.

26. Park H, Nakasato N, Tominaga T. Localization of abnormal discharges causing insular epilepsy by MEG. Tohoku J Exp Med. 2012;226(3):207-11.

27. Mohammed I, Gibbs A, Robert A, Bouthillier J, et al. The utility of MEG in presurgical evaluation of refractory insular epilepsy. Epilepsia. 2013;54(11):1950-9.

28. Jobst B, Gonzalez-Martinez J, Isnard J, Kahane P, et al. The insula and its epilepsies. Curr Rev Clin Sci. 2019;19(1):11-21.

29. Ius T, Pauletto G, Isola M, Gregoraci G, et al. Surgery for insular low-grade glioma: predictors of postoperative seizure outcome. J Neurosurg. 2014;120:12-23.

30. Ryvlin P, Kahane P. The hidden causes of surgery-resistant temporal lobe epilepsy: extratemporal or temporal plus? Curr Opin Neurol. 2005; 18(2):125-7.

31. Delev D, Oehl B, Steinhoff B, Nakagawa B, et al. Surgical treatment of Extratemporal epilepsy:results and prognostic factors. Neurosurg. 2019; 84:242-52.
32. Duffau H, Pallud J, Mandonnet E. Medically intractable epilepsy from insular low-grade gliomas: improvement after an extended lesionectomy. Acta Neurochir. 2002;144(6):563-72 discussion 572-573.

33. Guenot M, Isnard J, Ryvlin P, et al. SEEG guided RF thermocoagulation of epileptic foci. Epilepsia. 2004;45:1368-74.

34. Perry M, Donahue D, Malik S, Keator $C$, et al. Magnetic resonance imagingguided laser interstitial thermal therapy as treatment for intractable insular epilepsy in children. J Neurosurg Pediatr. 2017;20:575-82
Ready to submit your research? Choose BMC and benefit from:

- fast, convenient online submission

- thorough peer review by experienced researchers in your field

- rapid publication on acceptance

- support for research data, including large and complex data types

- gold Open Access which fosters wider collaboration and increased citations

- maximum visibility for your research: over $100 \mathrm{M}$ website views per year

At BMC, research is always in progress.

Learn more biomedcentral.com/submissions 